\title{
Higgs potential near-criticality from a de Sitter swampland-like condition
}

\author{
Jae-hyeon Park \\ Quantum Universe Center, Korea Institute for Advanced Study, 85 Hoegiro Dongdaemungu, \\ Seoul 02455, Republic of Korea
}

(Received 27 February 2019; published 19 June 2019)

\begin{abstract}
Motivated by the recently proposed de Sitter swampland conjecture, a formally same condition imposed instead on the convex and real exact effective potential is contemplated. Compared to the original conjecture, the modified condition admits a broader class of low-energy effective theories such as those with local maxima and/or false de Sitter vacua with some restrictions, as long as there is an anti-de Sitter vacuum. The observed accelerating expansion of the universe might therefore be attributed to a quintessence or a metastable vacuum. The former solution can be simplified and thus is better compatible with phenomenological constraints thanks to the convexity of the effective potential. Among the latter class of solutions is found the enthralling possibility that the modified condition is in fact behind the experimentally favored metastability of the Higgs potential with an instability scale below or around the Planck scale.
\end{abstract}

DOI: 10.1103/PhysRevD.99.116013

A quantum field theory (QFT) is said to belong to the landscape, if it is a low-energy effective field theory (EFT) of string theory. Otherwise, it is said to belong to the swampland $[1,2]$. These classifications were introduced as certain classes of theories could not be related to string theory despite enormous efforts. Motivated by such theoretical experience, criteria have been conjectured that are supposed to characterize EFTs in the landscape or swampland, see for a review [3].

In particular, the lack of any known rigorous construction of a de Sitter (dS) vacuum from string theory has recently led to the original version of the dS swampland conjecture [4],

$$
M_{\mathrm{Pl}}|\nabla V|>c V, \quad 0<c \sim \mathcal{O}(1),
$$

where $M_{\mathrm{Pl}}$ is the reduced Planck mass, $V$ is the scalar potential in the EFT, $\nabla V$ is its gradient with respect to the scalar fields with its norm defined using the metric on the field space. Indeed, this inequality says that $V$ at any extremum must be negative, thereby excluding any EFT with a dS extremum from the landscape. Still to keep our $\mathrm{dS}$ universe within the landscape, we are assumed to be living not at a (false) vacuum but on a nonvanishing slope of $V$ in the direction of some scalar field usually dubbed a quintessence $[5,6]$.

Published by the American Physical Society under the terms of the Creative Commons Attribution 4.0 International license. Further distribution of this work must maintain attribution to the author(s) and the published article's title, journal citation, and DOI. Funded by SCOAP ${ }^{3}$.
This conjecture has subsequently been refined to incorporate as an alternative condition the inequality $[7,8]$,

$$
M_{\mathrm{Pl}}^{2} \min \left(\nabla_{i} \nabla_{j} V\right) \leq-c^{\prime} V, \quad 0<c^{\prime} \sim \mathcal{O}(1),
$$

which bounds the minimum eigenvalue of the Hessian of $V$ in an orthonormal frame. (Other ways of refinement have also been proposed [9].) This refinement followed as difficulties had been encountered when the original conjecture (1) was applied to firmly established phenomenological particle physics models. The root of the troubles was that (1) forbids any local maximum of $V$ with a positive value which appears necessarily in EFTs with (spontaneous) symmetry breaking. Known instances include the center of the Higgs potential [10-13] as well as the local maxima found in the periodic potentials of the neutral pion [12] and the hypothetical QCD axion [11]. These counterexamples are admitted by the refined conjecture (1),(2). The swampland conjectures have also been put to the test in the context of cosmic inflation, to reveal differing degrees of compatibility with the models [14].

As the dS swampland conjectures restrict the scalar potential, a brief review of its different formulations should be in order. First of all, one can define the generating functional,

$$
Z[J]=\int \mathcal{D} \phi \exp \left\{i \int d^{4} x(\mathcal{L}[\phi(x)]+J(x) \phi(x))\right\},
$$

in terms of the classical Lagrangian $\mathcal{L}$ and the external currents $J$ as sources for the fields $\phi$. The connected generating functional is then given by 


$$
W[J]=-i \ln Z[J],
$$

of which the Legendre transform yields the one-particle irreducible effective action [15],

$$
\Gamma[\phi]=W[J]-\int d^{4} x J(x) \phi(x) .
$$

The effective potential can be defined as $\Gamma[\phi]$ specialized to coordinate-independent field expectation values [16],

$$
V_{\mathrm{np}}(\phi)=-\left.\frac{1}{V T} \Gamma[\phi]\right|_{\phi=\text { const. }},
$$

with the spacetime volume $V T$ factored out. This nonperturbatively defined exact effective potential $V_{\text {np }}$ can be approximated by a perturbation series in the form,

$$
V_{\mathrm{np}} \simeq V_{\mathrm{pert}} \equiv V_{0}+V_{1}+V_{2}+\cdots,
$$

where $V_{0}$ coincides with the tree-level potential in the classical Lagrangian $\mathcal{L}$, and $V_{1,2, \ldots}$ are the loop corrections at each order. It shall be understood that $V_{\text {pert }}$ may also include nonperturbative contributions as in the potential of an axion or mesons.

It is well known that $V_{\text {np }}$ and $V_{\text {pert }}$ are gauge [16] and renormalization scale dependent, but their values at the extrema are not [17] and are regarded as physical quantities, see for scale dependence e.g., $[18,19]$. This unphysical nature of effective potentials stems from the fact that their arguments i.e., the scalar field values are not physical quantities. In perturbation theory, the scale dependence of $V_{\text {pert }}$ is reduced as higher and higher order loops are included. This makes it mandatory to perform an all-order resummation of the large logarithms, when there is an orders-of-magnitude separation between the scale at which the input parameters are fixed and the scale at which $V_{\text {pert }}$ is to be evaluated.

Another remarkable property of $V_{\text {np }}$ (but not of $V_{\text {pert }}$ ) is its convexity [20]. This means especially that $V_{\text {np }}$ does not have any local maximum even if $V_{0}$ or $V_{\text {pert }}$ does. The shape of $V_{\text {np }}$ between the two local minima $\phi_{1}$ and $\phi_{2}$ of $V_{\text {pert }}$ is linear [21-23], and thus can be approximated by the linear interpolation (see e.g., [24,25]),

$$
\begin{aligned}
V_{\mathrm{np}}\left(x \phi_{1}+(1-x) \phi_{2}\right) & \simeq x V_{\text {pert }}\left(\phi_{1}\right)+(1-x) V_{\text {pert }}\left(\phi_{2}\right), \\
0 & <x<1,
\end{aligned}
$$

analogous to the Maxwell construction for free energies in thermodynamics, see e.g., [25]. This has also been a traditional way to resolve doubts about the imaginary part of $V_{\text {pert }}$ that develops at points where $V_{0}$ is concave $[16,21,23]$. The above construction is guaranteed to inherit the reality of $V_{\mathrm{np}}$ originating from its definition.
Recalling these properties of effective potentials, one might then ask a natural question: which $V$ does the dS swampland conjecture concern? In this work, the quantum limit of choosing $V_{\mathrm{np}}$ shall be entertained, in which case the original conjecture (1) would read

$$
M_{\mathrm{Pl}}\left|\nabla V_{\mathrm{np}}\right|>c V_{\mathrm{np}}, \quad 0<c \sim \mathcal{O}(1) .
$$

As has been pointed out recently [26], this modification weakens (1) to such an extent that the supplementary condition (2) would not be needed to accommodate the particle physics models with a local maximum in the potential [10-13]. Paraphrased in terms of $V_{\text {pert }}$, (9) does not prohibit any of its local maxima, dS or not, since they are all flattened in $V_{\mathrm{np}}$. For the same reason, it is obviously more permissive than the original (1) as well as the refined dS swampland conjectures (1),(2). Note that (2) becomes redundant, thereby rendering both versions of the conjectures equivalent, if $V$ therein is replaced by $V_{\mathrm{np}}$ [26]. A global minimum still needs to be negative.

An immediate consequence of (9) would therefore be that a dS space cannot be ascribed to a global minimum of $V_{\text {pert }}$. Given the astronomical observations indicating a small but positive vacuum energy, this would imply that we are not living in a true vacuum. There are two ways to negate the last noun phrase: (a) a false vacuum, or (b) a nonvacuum. Note that the former possibility has not been mentioned in Ref. [26].

Option (b) would mean that $\left|\nabla V_{\text {pert }}\right|>0$ in fact where we are living owing to a nonvanishing slope in the direction of an extra scalar field such as a quintessence. This bears a similarity to how a quintessence reconciles the original dS conjecture (1) with the cosmological constant [4]. A major difference is however that the local maximum of the perturbative Standard Model (SM) Higgs potential would not require a complicated interaction between the quintessence and the Higgs [10], as $V_{\text {np }}$ has no local maximum. It would therefore be enough to add quintessence terms simply to the Higgs potential, thereby eluding the constraints from a long-range force and time dependence of the proton-to-electron mass ratio [13].

With option (a), one can tell further details of the metastability with the aid of a "Maxwell construction." First, imagine a perturbative potential having only two minima which are connected by the scalar field direction $\phi$. Replacing $V_{\text {np }}$ between these two minima by (8) lets (9) be represented by a pair of inequalities,

$$
\begin{gathered}
V_{\text {pert }}\left(\phi_{+}\right)<\frac{M_{\mathrm{Pl}}}{c}\left|\frac{V_{\text {pert }}\left(\phi_{+}\right)-V_{\text {pert }}\left(\phi_{-}\right)}{\phi_{+}-\phi_{-}}\right|, \\
V_{\text {pert }}\left(\phi_{-}\right)<0,
\end{gathered}
$$

where $\phi_{ \pm}$are the field values at our false and the true vacua, respectively. These conditions are depicted in the left panel 

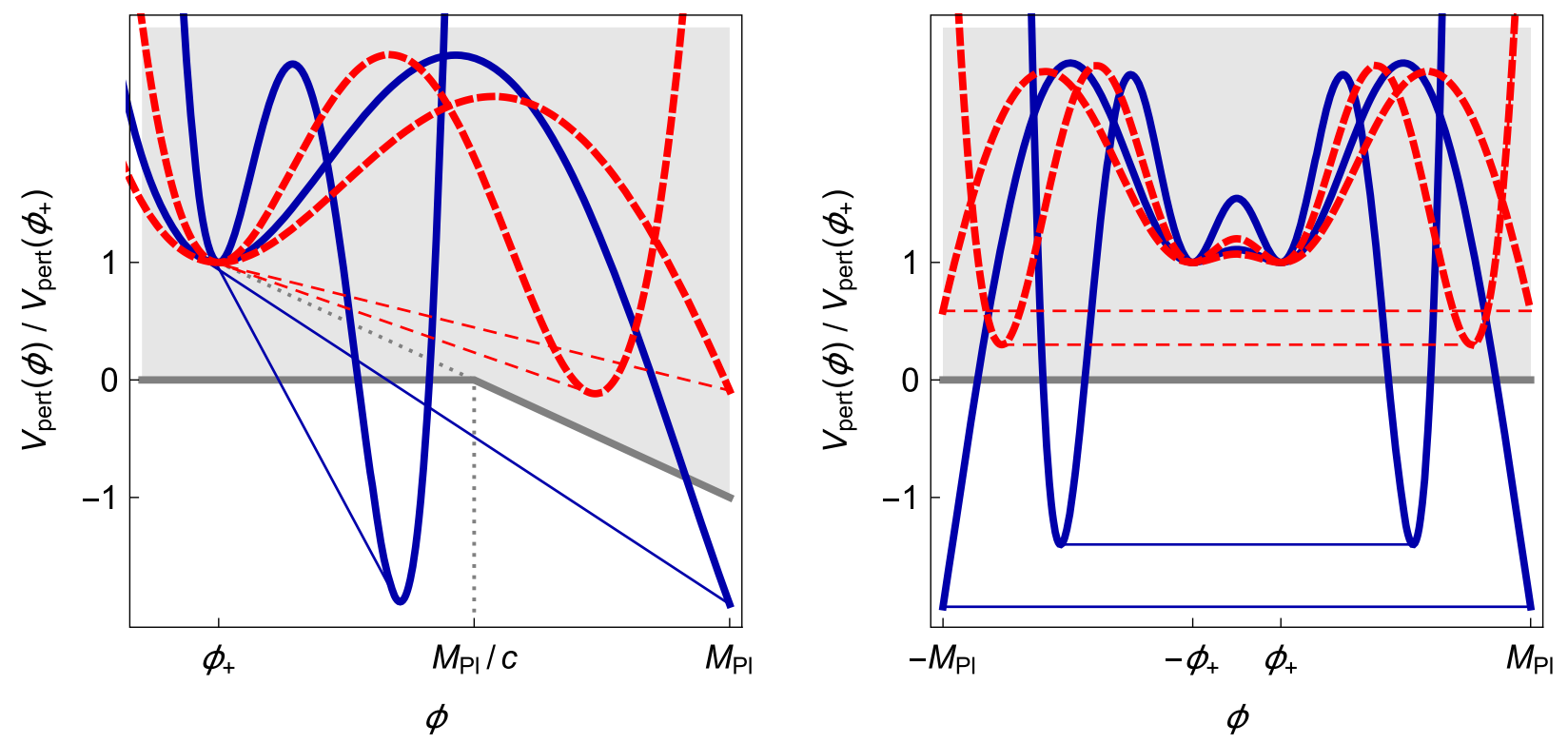

FIG. 1. Schematic shapes of perturbative scalar potentials (thick) allowed (solid) or disallowed (dashed) by (10) and the corresponding "Maxwell constructions" (thin), for $c>1$. The global minima of each potential cannot lie in the light grey region. For $c<1$, the horizontal part of the grey line in the left panel would extend up to the right border.

of Fig. 1, where $c$ is assumed to be larger than unity as suggested by some reported lower bounds on $|\nabla V| / V$ in type IIA/B compactifications [4]. Essentially, the global minimum $V_{\text {pert }}\left(\phi_{-}\right)$is constrained to be firstly negative and then deep enough to meet the lower bound on $\left|\nabla V_{\mathrm{np}}\left(\phi_{+}\right)\right|$. It might happen that $V_{\text {pert }}\left(\phi_{-}\right)$is at the end of the validity range of QFT which is assumed to be $M_{\mathrm{Pl}}$ based on the distance conjecture [2]. In such a case, (10b) would not apply as the slope does not vanish at $\phi_{-}$. If the cutoff on $|\phi|$ is smaller than $M_{\mathrm{Pl}} / c$, there would be another logical possibility that $V_{\text {pert }}(\phi)$ does not necessarily need to cross the grey line. It would only need to cross the slanted grey dotted line and then hit the cutoff at a nonvanishing slope. Numerically, $V_{\text {pert }}\left(\phi_{+}\right) \sim \mathcal{O}\left(\mathrm{meV}^{4}\right)$ would be in most cases negligible compared to typical scales characterizing $V_{\text {pert }}$ such as the width and height of its barrier. Therefore, the grey upper bound on $V_{\text {pert }}\left(\phi_{-}\right)$with a mild kink would be approximated excellently by a straight horizontal line in the left panel of Fig. 1.

A reflection symmetry of the potential would simplify the scrutiny as illustrated in the right panel of Fig. 1. The "Maxwell construction" would then be a constant between the two global minima which (9) would require to be negative. In this case, $V_{\text {pert }}(\phi)$ would need to cross zero within the validity range of QFT, whether the slope vanishes at the global minima or not.

Subdividing option (a), the metastability of our electroweak vacuum might be attributed to (a1) a new direction in the field space, or (a2) the SM Higgs. Once an extra field is admitted as in case (a1), it is straightforward to build a model with metastability and there are already many such examples as: supersymmetry with charge and/or color breaking minima [27-29], metastable supersymmetry breaking sectors [30], relaxion mechanism [31], scalar extensions of the Higgs sector [32], among others. In models with a stable Higgs potential, metastability would thus serve as a hint on the vacuum structure altered by the additional fields.

The remaining most predictive scenario would be case (a2) without any extra field responsible for the metastability. This might be because there are no extra scalars at all or none of them induces a deeper minimum. An obvious but fascinating implication would then be that the perturbative SM Higgs potential is destined to be metastable.

In this case, a more concrete statement can be made in the context of the SM. Assuming that $\phi_{-} \gg \phi_{+} \approx$ $246 \mathrm{GeV}$, one can use the renormalization group (RG) improved tree-level potential, $V_{\text {pert }}\left(\phi_{-}\right)=\lambda\left(\phi_{-}\right) \phi_{-}^{4} / 4$, with the running quartic Higgs coupling $\lambda(\mu)$ at the renormalization scale $\mu=\phi_{-}$. The preceding discussions about condition (9) on symmetric potentials would then lead to the requirement that $\lambda(\mu)$ turn negative below or around $M_{\mathrm{Pl}}$, an upper limit due to the distance conjecture [2] as well as the inherent ultraviolet cutoff of the EFT within which the $\beta$-function of $\lambda$ is computed.

It is amusing to notice that this prediction of an instability scale below or around $M_{\mathrm{Pl}}$ is pleasantly consistent with the already favored interpretation of the experimental data, even though it is demanded independently of the low-energy boundary conditions on the running couplings. The RG evolution of $\lambda$ has been analyzed employing higher order corrections at the accuracy of 2-loop matching at the weak scale plus 3-loop running 
up to high scales $[19,33,34]$. A first resulting feature to notice is that $\lambda(\mu)$ maintains a single minimum, positive or negative, around $10^{17-18} \mathrm{GeV}$ while the top quark and Higgs masses as well as the strong coupling constant are varied by $\pm 3 \sigma$ [33]. The upper end of this range would therefore bound a zero of $\lambda(\mu)$ from above, if it exists. Then, the central values of the SM parameters point to metastability of the Higgs potential with $\lambda(\mu)$ crossing zero at a scale around $\mu \sim 10^{10} \mathrm{GeV}$, which can vary between $10^{8}$ and $10^{18} \mathrm{GeV}$ if $3 \sigma$ uncertainties in the data are taken into account. Additional ambiguity in the instability scale arising from its gauge dependence has been investigated numerically [35].

There have been attempts to understand this intriguing selection of a special point in the parameter space: there might be an underlying theory which brings the SM to that particular point via the matching conditions [29,36]; nearcriticality might be an attractor within the multiverse [37]. Yet another inspiring possibility would be that inequality (9) is in fact the reason behind the metastability of the Higgs potential, if the condition has relevance to physical laws of nature such as the still developing theory of quantum gravity.

It is hard to judge whether (9) has something to do with string theory or not. As it turns out, the condition is at variance with the original motivation for (1),(2), i.e., to exclude dS minima. On the contrary, (9) admits false dS vacua albeit with restrictions on $\left|\nabla V_{\mathrm{np}}\right| / V_{\mathrm{np}}$. Neither has it been proved however that the landscape contains no dS vacua. From the field theoretic point of view, the use of $V_{\mathrm{np}}$ in conjecture (1) has been advocated [26], emphasizing the necessity of large scale nonperturbative scalar field fluctuations for a consistent low-energy description of the theory. In particular, it is not clear how to interpret the dS criteria as imposed on $V_{\text {pert }}$ if it has an imaginary part. This doubt would be naturally resolved by employing $V_{\mathrm{np}}$ instead which is real-valued by definition. In any case, one might make at least the following conservative statements. It is a natural extrapolation of (1),(2) to incorporate into $V$ therein as many quantum effects as there are. The criterion thus modified is less restrictive than the original but not trivial either, and furthermore suggests intriguing phenomenological scenarios including a potential solution to a big puzzle raised by accelerator physics.

To sum up, a theoretical constraint on quantum field theoretic models has been considered. Its form is identical to the original dS swampland conjecture except that the effective potential is assumed to integrate all possible (non)perturbative quantum effects, thereby guaranteeing its reality. Due to the convexity of the exact effective potential $V_{\mathrm{np}}$, the modified condition is more permissive than the original as well as the refined conjectures. Specifically, it accepts local maxima and false dS vacua in the perturbative potential $V_{\text {pert }}$ as long as the slope of $V_{\text {np }}$ is everywhere steep enough, although the true vacua must still be anti-de Sitter. This naturally resolves conflicts with the essential local maxima in established particle physics models. Moreover, it opens up the possibility of attributing the observed positive cosmological constant not only to a quintessence but also to a metastable dS vacuum. In the former case, the quintessence might avoid phenomenological obstacles thanks to its simpler interactions with the Higgs. In the latter case, the Higgs potential would need to have a deeper minimum unless the metastability is caused by an extra scalar field. The Higgs instability scale is then predicted to be below or around $M_{\mathrm{Pl}}$, from the distance conjecture plus the ultraviolet cutoff of the EFT. This might shed light on the well known near-critical structure of the SM Higgs potential for the preferred values of low-energy data including the top quark and Higgs masses.

\section{ACKNOWLEDGMENTS}

The author thanks Jason Evans, Lucien Heurtier, JongChul Park, Seodong Shin, and Seokhoon Yun, for the informative and encouraging discussions. This work has been highly boosted by the focus research program "Dark Matter as a Portal to New Physics" supported by the Asia Pacific Center for Theoretical Physics.
[1] C. Vafa, arXiv:hep-th/0509212.

[2] H. Ooguri and C. Vafa, Nucl. Phys. B766, 21 (2007).

[3] T. D. Brennan, F. Carta, and C. Vafa, Proc. Sci. TASI2017 (2017) 015.

[4] G. Obied, H. Ooguri, L. Spodyneiko, and C. Vafa, arXiv: 1806.08362.

[5] B. Ratra and P. J. E. Peebles, Phys. Rev. D 37, 3406 (1988); C. Wetterich, Nucl. Phys. B302, 668 (1988); I. Zlatev, L. M. Wang, and P. J. Steinhardt, Phys. Rev. Lett. 82, 896 (1999).
[6] S. Tsujikawa, Classical Quantum Gravity 30, 214003 (2013).

[7] S. K. Garg and C. Krishnan, arXiv:1807.05193.

[8] H. Ooguri, E. Palti, G. Shiu, and C. Vafa, Phys. Lett. B 788, 180 (2019).

[9] G. Dvali and C. Gomez, Fortschr. Phys. 67, 1800092 (2019); D. Andriot, Phys. Lett. B 785, 570 (2018); D. Andriot and C. Roupec, Fortschr. Phys. 67, 1800105 (2019).

[10] F. Denef, A. Hebecker, and T. Wrase, Phys. Rev. D 98, 086004 (2018). 
[11] H. Murayama, M. Yamazaki, and T. T. Yanagida, J. High Energy Phys. 12 (2018) 032.

[12] K. Choi, D. Chway, and C. S. Shin, J. High Energy Phys. 11 (2018) 142.

[13] K. Hamaguchi, M. Ibe, and T. Moroi, J. High Energy Phys. 12 (2018) 023.

[14] W. H. Kinney, S. Vagnozzi, and L. Visinelli, Classical Quantum Gravity 36, 117001 (2019); C. Han, S. Pi, and M. Sasaki, Phys. Lett. B 791, 314 (2019); S. J. Wang, Phys. Rev. D 99, 023529 (2019); S. C. Park, J. Cosmol. Astropart. Phys. 01 (2019) 053; D. Y. Cheong, S. M. Lee, and S. C. Park, Phys. Lett. B 789, 336 (2019); M. S. Seo, Phys. Rev. D 99, 106004 (2019).

[15] G. Jona-Lasinio, Nuovo Cimento 34, 1790 (1964).

[16] R. Jackiw, Phys. Rev. D 9, 1686 (1974).

[17] N. K. Nielsen, Nucl. Phys. B101, 173 (1975); R. Fukuda and T. Kugo, Phys. Rev. D 13, 3469 (1976).

[18] A. Andreassen, W. Frost, and M. D. Schwartz, Phys. Rev. D 91, 016009 (2015).

[19] A. Andreassen, W. Frost, and M. D. Schwartz, Phys. Rev. Lett. 113, 241801 (2014).

[20] K. Symanzik, Commun. Math. Phys. 16, 48 (1970).

[21] Y. Fujimoto, L. O'Raifeartaigh, and G. Parravicini, Nucl. Phys. B212, 268 (1983).

[22] R. W. Haymaker and J. Perez-Mercader, Phys. Rev. D 27, 1948 (1983); C. M. Bender and F. Cooper, Nucl. Phys. B224, 403 (1983); M. Hindmarsh and D. Johnston, J. Phys. A 19, 141 (1986); V. Branchina, P. Castorina, and D. Zappala, Phys. Rev. D 41, 1948 (1990).

[23] K. E. Cahill, Phys. Rev. D 52, 4704 (1995).

[24] S. Weinberg, The Quantum Theory of Fields (Cambridge University Press, Cambridge, England, 1996), Vol. 2, Sec. 16.3.

[25] M. E. Peskin and D. V. Schroeder, An Introduction to Quantum Field Theory (Westview Press, Boulder, 1995), Sec. 11.3.
[26] A. Kobakhidze, arXiv:1901.08137.

[27] M. Claudson, L. J. Hall, and I. Hinchliffe, Nucl. Phys. B228, 501 (1983).

[28] M. Kakizaki, E. K. Park, J.-h. Park, and A. Santa, Phys. Lett. B 749, 44 (2015); M. Bach, J.-h. Park, D. Stöckinger, and H. Stöckinger-Kim, J. High Energy Phys. 10 (2015) 026.

[29] J.-h. Park, arXiv:1809.07774.

[30] K. A. Intriligator, N. Seiberg, and D. Shih, J. High Energy Phys. 04 (2006) 021.

[31] P. W. Graham, D. E. Kaplan, and S. Rajendran, Phys. Rev. Lett. 115, 221801 (2015).

[32] F. Staub, Phys. Lett. B 776, 407 (2018).

[33] G. Degrassi, S. Di Vita, J. Elias-Miro, J. R. Espinosa, G. F. Giudice, G. Isidori, and A. Strumia, J. High Energy Phys. 08 (2012) 098; D. Buttazzo, G. Degrassi, P. P. Giardino, G. F. Giudice, F. Sala, A. Salvio, and A. Strumia, J. High Energy Phys. 12 (2013) 089.

[34] A. V. Bednyakov, B. A. Kniehl, A. F. Pikelner, and O. L. Veretin, Phys. Rev. Lett. 115, 201802 (2015).

[35] L. Di Luzio and L. Mihaila, J. High Energy Phys. 06 (2014) 079; D. Boyanovsky, W. Loinaz, and R. S. Willey, Phys. Rev. D 57, 100 (1998); J. L. Cook, L. M. Krauss, A. J. Long, and S. Sabharwal, Phys. Rev. D 89, 103525 (2014).

[36] L. J. Hall and Y. Nomura, J. High Energy Phys. 03 (2010) 076; G. F. Giudice and A. Strumia, Nucl. Phys. B858, 63 (2012); M. E. Cabrera, J. A. Casas, and A. Delgado, Phys. Rev. Lett. 108, 021802 (2012); A. Arbey, M. Battaglia, A. Djouadi, F. Mahmoudi, and J. Quevillon, Phys. Lett. B 708, 162 (2012); L. E. Ibanez and I. Valenzuela, J. High Energy Phys. 05 (2013) 064; A. Hebecker, A. K. Knochel, and T. Weigand, Nucl. Phys. B874, 1 (2013); M. Ibe, S. Matsumoto, and T. T. Yanagida, Phys. Lett. B 732, 214 (2014); B. C. Allanach and A. Voigt, Eur. Phys. J. C 78, 573 (2018).

[37] G. F. Giudice and R. Rattazzi, Nucl. Phys. B757, 19 (2006). 